\title{
O papel da avaliação para a tomada de decisão na gestão de serviços de saúde
}

\author{
The role of evaluation in decision-making \\ in the management of health services
}

Oswaldo Yoshimi Tanaka ${ }^{1}$

Edson Mamoru Tamaki ${ }^{2}$

${ }^{1}$ Faculdade de Saúde Pública, Universidade de São Paulo. Av. Dr. Arnaldo 715, Cerqueira César. 01246-904 São Paulo SP. oytanaka@usp.br ${ }^{2}$ Departamento de Saúde Coletiva, Centro de Ciências Biológicas e da Saúde, Universidade Federal de Mato Grosso do Sul
Abstract The management of health services is a complex administrative practice due to the breadth of the field of health and the need to reconcile individual, corporate and collective interests that are not always convergent. In this context, the evaluation needs to have specific characteristics in order to fulfill its role. The scope of this study was to establish the characteristics that the evaluation for the management of health services should have to contribute to decision-making. Usefulness, opportunity, feasibility, reliability, objectivity and directionality represent the set of principles upon which the evaluation should be based. Evaluations should lead to decisions that guarantee not only their efficiency and effectiveness but also their implementation. The evaluation process should ensure that decisions involve all stakeholders in order to render the implementation of decisions feasible, and take into account the health needs of the population and the goals set for the services. The scope of this article is to elicit a debate among different stakeholders in the evaluation in the hope that it can contribute to the reflection on the real usefulness of evaluations in which the political component in management has been increasingly prevalent.

Key words Evaluation of health services, Management of health services, Decision-making, Evaluation process
Resumo A gestão de serviços de saúde é uma prática administrativa complexa em função da amplitude desse campo e da necessidade de conciliar interesses individuais, corporativos e coletivos nem sempre convergentes. Nesse contexto, a avaliação necessita ter características particulares para poder cumprir com o seu papel. O objetivo deste estudo foi o de estabelecer as características que a avaliação para a gestão de serviços de saúde deve possuir para contribuir para a tomada de decisão. Utilidade, oportunidade, factibilidade, confiabilidade, objetividade e direcionalidade constituem o conjunto de princípios que deve ancorar essa avaliação. Uma avaliação que conduza a decisões que garantam não somente a sua eficiência e eficácia, mas também a sua implementação. O processo avaliativo deve buscar que as decisões tomadas levem em consideração as necessidades de saúde da população, as metas definidas pelos serviços e que envolvam todos os interessados de forma a tornar viável a implementação das decisões tomadas. $O$ presente artigo tem o propósito de desencadear um debate entre os distintos interessados em avaliação na expectativa de que possam contribuir na reflexão quanto à real utilidade da avaliação em que o componente político na gestão tem sido cada vez mais predominante.

Palavras-chaves Avaliação de serviços de saúde, Gestão de serviços de saúde, Tomada de decisão, Processo avaliativo 


\section{Introdução}

A gestão de serviços de saúde constitui uma prática administrativa que tem a finalidade de otimizar o funcionamento das organizações de forma a obter o máximo de eficiência (relação entre produtos e recursos empregados), eficácia (atingir os objetivos estabelecidos) e efetividade (resolver os problemas identificados). Nesse processo o gestor utiliza conhecimentos, técnicas e procedimentos que lhe permitem conduzir o funcionamento dos serviços na direção dos objetivos definidos.

A avaliação constitui um instrumento essencial de apoio à gestão pela sua capacidade de melhorar a qualidade da tomada de decisão. Apesar disso, o seu uso ainda é incipiente na gestão de serviços de saúde ${ }^{1-3}$.

Um obstáculo para uma utilização mais ampla da avaliação na tomada de decisão nos serviços de saúde é que a sua implementação requer recursos e tempo, o que dificulta a sua utilização para problemas que necessitem de soluções imediatas. Nessas situações, que são frequentes quando se trata da saúde de pessoas e da população, somente a existência de um conhecimento acumulado, decorrente de avaliações passadas ou previamente planejadas, pode contribuir para a tomada de decisão.

A capacidade da avaliação de contribuir para o aperfeiçoamento do processo de decisão na saúde se confronta com a complexidade do campo, caracterizado pelos múltiplos fatores que condicionam a saúde e a doença, o que faz com que a sua construção seja revestida de características e se paute em princípios próprios para produzir os resultados esperados na saúde da população.

\section{Avaliação e o processo de gestão}

A avaliação pode ser referida como um conhecimento produzido no campo teórico-metodológico aplicável a um objeto quando há a necessidade de emitir um julgamento de valor, independentemente do uso que será feito do seu produto. Essa concepção de avaliação cria um campo próprio de conhecimento para avaliação, aumentando a sua interface com o campo da pesquisa ${ }^{4}$.

No entanto, a maior parte das definições de avaliação se apresenta de forma indissociável ao processo de tomada de decisão ou à sua aplicação no campo da gestão. Essa observação é corroborada por Matida e Camacho ${ }^{5}$ que, analisando o contexto das pesquisas de avaliação, constatam que elas conferem à avaliação, quer seja ela administrativa quer seja científica, um objetivo - subsidiar a tomada de decisão. A aplicabilidade do ato de avaliar integra o seu conceito.

A gestão envolve a melhoria do funcionamento das organizações e para isso ela tem que encontrar a melhor combinação possível dos recursos disponíveis para atingir os seus objetivos. Encontrada essa combinação, ela é institucionalizada através da formalização de estruturas, processos, rotinas, fluxos e procedimentos.

Toda essa construção é concebida visando obter um funcionamento otimizado em um contexto complexo, caracterizado pelo conjunto de fatores que condicionam a saúde e a doença e que produz, permanentemente, situações não previsíveis que exigem a tomada de decisões. Há a necessidade de dar respostas a essas situações a fim de manter o funcionamento ou melhorar os níveis de eficiência e eficácia dos serviços.

Avaliar consiste fundamentalmente em fazer um julgamento de valor a respeito de uma intervenção ou sobre qualquer um dos seus componentes com o objetivo de ajudar na tomada de decisã $0^{6}$. Nesse sentido, o papel da avaliação no processo de gestão é o de fornecer elementos de conhecimento que subsidiem a tomada de decisão, propiciando o aumento da eficiência, eficácia e efetividade das atividades desenvolvidas pelo serviço ou pela organização.

A tomada de decisão é uma responsabilidade e uma competência formal do gestor que, além das informações obtidas no processo avaliativo, utiliza o conhecimento pessoal que possui (referências técnicas, políticas, institucionais, sociais, culturais entre outras) ou a percepção que tem do problema, forma uma convicção e toma uma decisão, mobilizando recursos necessários.

A tomada de decisão na gestão em saúde é complexa e permeada de subjetividade e incertezas. Paim e Teixeira ${ }^{7}$, referindo-se à área de políticas, planejamento e gestão em saúde, ressaltam essas características afirmando que: há momentos em que faltam conhecimentos para a tomada de decisões, outros em que há conhecimentos suficientes, mas as decisões são adiadas e, ainda, existem aqueles em que as decisões são necessárias mesmo diante de escassas evidências.

\section{A Avaliação para a Gestão de Serviços de Saúde - AGSS}

Abordar a gestão de serviços de saúde constitui um desafio, pois os serviços de saúde são estruturas organizacionais complexas extremamen- 
te diversificadas, constituídas por profissionais e tecnologias organizados para a atenção à saúde da população. Por outro lado, os serviços de saúde constituem objetos que desafiam categorizações $e$ dificultam as análises que buscam identificar $e$ mensurar a sua participação e o seu impacto sobre os problemas de saúde de populações especificadas ${ }^{2}$.

A gestão dos serviços de saúde tem que levar em consideração tanto questões internas - organização e funcionamento do serviço - como questões externas - o seu papel no sistema de saúde e o impacto na saúde da população. É um papel próprio da área de políticas, planejamento e gestão que, no âmbito da saúde coletiva, expressa com mais clareza a dupla dimensão (saber e práticas) do campo, ou seja, o fato de que o conhecimento produzido responde a problemas e desafios colocados pelos sujeitos em sua ação política em determinados contextos históricos ${ }^{7}$.

\section{Caracterização da Avaliação}

\section{para a Gestão de Serviços de Saúde}

A avaliação para a gestão de serviços de saúde é um processo técnico-administrativo e político de julgamento do valor ou mérito de algo, para subsidiar a tomada de decisão no cotidiano, o que significa produzir informações capazes de apoiar uma intervenção de forma oportuna ${ }^{8}$. É baseada na utilização de métodos e técnicas de pesquisa na sua concepção, formulação e implementação.

A AGSS tem origem no momento em que há a identificação de uma situação circunscrita, identificada como problema, a partir da definição do propósito. Isto significa que a avaliação deve ser compreendida como um instrumento ou ferramenta da gestão para enfrentar e resolver um problema do serviço ou do programa de saúde, ou seja, tem por finalidade propiciar um processo de decisão oportuno no tempo, com confiabilidade e abrangência de informações, segundo objetivos das distintas audiências ${ }^{8}$.

A avaliação deve ter a objetividade necessária para tornar o processo factível dentro do tempo e dos recursos disponíveis, mesmo que para isso seja necessário simplificar processos, limitar a profundidade do estudo e relativizar a precisão e abrangência dos resultados, preservando o que lhe é essencial: a contribuição para a tomada de decisão e para a sua implementação.

\section{Princípios da Avaliação}

para a Gestão de Serviços de Saúde

Para estruturar um processo avaliativo que tenha a capacidade de atingir os objetivos da AGSS é necessário que um conjunto de princípios seja levado em consideração: a) Utilidade. A avaliação para a gestão de serviços de saúde tem como fundamento central a sua utilidade no funcionamento dos serviços de saúde. Ela tem que subsidiar tomadas de decisão que tragam soluções aos problemas dos serviços que estão impedindo atingir os objetivos fixados ou que estão dificultando a obtenção de um maior impacto na saúde da população.

b) Oportunidade. A avaliação tem que ser feita em tempo para que os seus resultados possam ser utilizados na tomada de decisão, ou seja, o prazo para a sua execução vai ser o tempo existente entre a demanda da avaliação e o momento da tomada de decisão.

c) Factibilidade. A avaliação tem que ser não somente viável em termos técnicos, econômicos e políticos, mas também tem que ter a capacidade de produzir os efeitos esperados das decisões tomadas, ou seja, tem que ser pragmática em termos de fazer escolhas viáveis e sobre as quais tenha governabilidade.

d) Confiabilidade. Como um processo que visa subsidiar tanto a tomada de decisão como a sua implementação, a avaliação tem que ser revestida de racionalidade, coerência e consistência a fim de que ela possa ser considerada válida e aceita por todos aqueles que estão envolvidos na decisão e na sua execução.

e) Objetividade. Considerando a existência de limites para a sua realização, a avaliação para a gestão deve buscar o melhor conhecimento e o maior aprofundamento possível dentro do tempo e dos recursos disponíveis. Isso significa que, nessas condições, a obtenção da maior contribuição possível para a tomada de decisão é mais importante que a busca de uma maior validade da avaliação.

f) Direcionalidade. A avaliação para a gestão de serviços de saúde tem que conduzir, em todos os espaços possíveis, as escolhas na direção da resolução dos problemas que deram origem ao processo avaliativo, da satisfação das necessidades da população e da implementação das políticas do setor.

\section{Delineamento da Avaliação para a Gestão de Serviços de Saúde}

O fornecimento de informação em tempo hábil para o gestor é imprescindível para a melhoria qualitativa das decisões. Se houver um conhecimento acumulado de pesquisas, estudos e avaliações passadas, o procedimento adequado consiste na apropriação desse conhecimento e a sua utilização no processo de decisão. Se não 
houver, faz-se necessário estruturar um processo avaliativo o mais consistente possível dentro do tempo e dos recursos disponíveis.

$\mathrm{Na}$ rotina dos serviços de saúde a necessidade de resolver problemas de saúde da população ou de satisfazer às demandas de usuários limitam o tempo para a tomada de decisão, pois é necessário dar respostas às situações que já estão sendo vivenciadas pelo gestor. Isso faz com que as avaliações tendam: a ser executadas com rapidez; a utilizar as informações e os conhecimento já disponíveis; a utilizar instrumentos metodológicos que se adéquem ao tempo e recursos existentes; a reduzir e simplificar os elementos a serem estudados; e a utilizar um grau de precisão (nível de confiança) dos resultados suficiente para a decisão a ser tomada.

Ao discutir a avaliação como instrumento de gestão no contexto de programas e serviços de saúde, é preciso definir quem toma decisões. Sem a identificação de quem participa do processo decisório, a avaliação se torna apenas um diagnóstico de uma dada situação e não contribuirá para a modificação da situação avaliada. (...). Nesse sentido, o julgamento de valor resultante da avaliação deverá ter uma audiência claramente identificada, pois esta atua como sustentadora (stakeholders) não só do processo de avaliação, como das decisões que serão modificadas ou mantidas a partir de tais resultados ${ }^{8}$. A tomada de decisão não basta para resolver um problema, pois somente com a execução das ações decorrentes da decisão é que esse objetivo será atingido.

O envolvimento da audiência ganha uma importância estratégica. A AGSS deve privilegiar a construção de um processo avaliativo onde todos aqueles que participam ou que serão afetados pelas ações desencadeadas pela tomada e implementação da decisão, tornem-se partícipes e interessados pelos resultados da avaliação. Esse interesse será a garantia do uso da avaliação na gestão dos serviços de saúde que, para evitar distorções, isenção e aceitação deve ser participativo, democrático e não hierarquizado ${ }^{1}$.

A concepção do processo avaliativo tem que estar sintonizada com o contexto do objeto a ser avaliado, com as necessidades de saúde da população, com as escolhas do problema de saúde, com a identificação dos atores que têm poder de tomar as decisões, com os que têm a capacidade técnica de implementá-las e com as políticas, estratégias e prioridades do setor saúde. $\mathrm{O}$ avaliador tem de conceber um processo avaliativo através da escolha de metodologias, indicadores e de parâmetros que contemple essa diversidade de aspectos e que direcione as decisões e as ações para propiciar o máximo de benefício à saúde da população. Uma tarefa que faz com que cada avaliação seja um caso particular que requer criatividade por parte do investigador/avaliador na formulação da melhor estratégia, na seleção da abordagem, na definição de níveis e atributos, bem como na seleção de critérios, indicadores e padrões?.

\section{Formulação do objetivo}

e condução da avaliação

Sem uma clara pergunta avaliativa, como ponto de partida, não se produz uma avaliação e sim um diagnóstico ${ }^{10}$. No âmbito da AGSS essa afirmação ganha importância, pois a falta de clareza na formulação da pergunta avaliativa propiciará o acúmulo de dados e de informações que poderão melhorar o conhecimento do problema e do objeto de avaliação, mas não desencadeará uma tomada de decisão que venha alterar a situação de saúde da população.

O conhecimento do contexto da avaliação é essencial para estabelecer o seu objetivo, o que requer não apenas a identificação de perguntas e formulação de hipóteses, mas, antes, a verificação do conjunto de atores envolvidos, dos recursos disponíveis, do grau de complexidade do problema e das ações e, em particular, a concordância de perspectivas entre quem pleiteia a avaliação e quem a executa ${ }^{5}$.

Esse conhecimento é necessário para que a avaliação seja viável e tenha a capacidade de enfrentar o que Contandriopoulos cita como o grande desafio da avaliação: conseguir incorporar nas suas estratégias os pontos de vista de atores em diferentes posições, a fim de fornecer às instâncias de decisão as informações de que precisam para fazer um julgamento o mais amplo possivel ${ }^{1}$.

A avaliação tem que convergir para as necessidades da audiência da avaliação, ou seja, não só aqueles que são responsáveis pela tomada de decisão, mas também aqueles que serão responsáveis pela implementação das ações decorrentes da decisão tomada. Pelo fato de constituir uma cadeia de eventos, se um deles não executar a tarefa que lhe é devida a ação não será concretizada.

O produto dos serviços de saúde constitui algo que é parte integrante do usuário do serviço de saúde $e^{10}$, ou seja, se não houver a utilização dos serviços pela população não há a produção de saúde. Sob essa perspectiva, se evidencia a importância de ir ao encontro das necessidades de saúde e quem melhor pode expressá-las é a própria população. Portanto, cabe ao avaliador a responsabilidade de incorporar direta ou indiretamente a população-alvo na formulação do pro- 
cesso avaliativo, particularmente nas fases de identificação dos problemas de saúde, de formulação da pergunta avaliativa e na definição dos indicadores e parâmetros a serem utilizados.

O papel principal do avaliador será o de imprimir uma direcionalidade à decisão e de construir a viabilidade da sua implementação. Na viabilização da implementação da decisão a direcionalidade poderá ser dada por meio do envolvimento não somente dos que tomam as decisões mas também dos que as implementam e dos que são beneficiados por essas decisões.

\section{Estabelecimento de indicadores e parâmetros}

Os indicadores são variáveis que visam fornecer a melhor imagem possível de um objeto, no entanto, considerando as inúmeras facetas com que ele pode ser observado, podem existir inúmeros conjunto de indicadores que poderão formar essa imagem. Em face desse rol de possibilidades, a escolha dos indicadores que serão utilizados em uma avaliação constitui uma das tarefas críticas do avaliador.

Segundo a Organização Mundial da Saúde ${ }^{11}$, na escolha de um indicador deve ser levada em consideração a sua validade (medir efetivamente o que pretende medir), a sua fiabilidade (apresentar o mesmo resultado mesmo que seja utilizado por pessoas ou em circunstâncias diferentes), a sua sensibilidade (capacidade de captar mudanças na situação ou no objeto estudado), e sua especificidade (refletir mudanças apenas no objeto que está sendo estudado). Em função da aplicação que será feita dos indicadores, a esses atributos podem ser agregados outros como os citados pela RIPSA (Rede Interagencial de Informações para a Saúde) ${ }^{12}$ : mensurabilidade (basear-se em dados disponíveis ou fáceis de conseguir); relevância (responder a prioridades de saúde); e custo-efetividade (os resultados justificam o investimento de tempo e recursos).

Para atingir os objetivos estabelecidos, os indicadores a serem utilizados na AGSS também requererem alguns requisitos particulares:

a) ter a capacidade de dar resposta à pergunta avaliativa;

b) ter um poder de síntese, isto é, ... a maior capacidade possível de descrever e permitir a análise do fenômeno, ou das relações deste com os outros eventos do serviço ou do programa de saúde ${ }^{8}$;

c) ser factível, ou seja, passível de ser obtido em tempo para subsidiar o processo de decisão, respeitadas as limitações de tempo e recursos existentes para esse fim; d) ter a capacidade de mobilizar recursos, ou seja, sem esse atributo o indicador não terá o efeito de mobilizar aqueles que têm o poder de decidir e implementar as ações;

e) constituir um conjunto limitado de indicadores (03 a 05 indicadores para cada pergunta avaliativa) a fim de viabilizar a sua obtenção, a análise e a compreensão pelos gestores e demais interessados na avaliação;

f) ter aceitabilidade pela audiência a fim de que os seus resultados sejam legitimados e utilizados pelos gestores e técnicos envolvidos na ação; e

g) possuir uma parâmetro correspondente pois para se chegar ao juízo de valor, que é o pressuposto da avaliação, é imprescindível a comparação com parâmetros definidos. Para isso é necessário que, ao escolher os indicadores, se defina claramente com quê estes serão comparados ${ }^{13}$.

O conjunto de indicadores escolhidos deverá refletir fidedignamente o objeto e contemplar adequadamente a finalidade e os objetivos da avaliação.

O campo da avaliação em saúde conta com referenciais teórico-metodológicos e instrumentais de pesquisa que, como em qualquer outro campo de conhecimento, são passiveis de direcionamentos intencionais. Moraes, citado por Santos-Filho ${ }^{14}$ ressalta que os indicadores refletem $o$ sistema de valores do profissional que os constrói, o que implica em que formule certas hipóteses e perguntas e não outras. Nesse sentido, cabe ao avaliador fazer com que as políticas, os princípios e as estratégias institucionais (em contraposição aos pessoais) sejam contemplados no momento da concepção do processo avaliativo.

O poder de escolha dos critérios, parâmetros e indicadores de avaliação faculta ao avaliador uma capacidade de direcionar o processo avaliativo para decisões que garantam não somente a pertinência, a coerência e a consistência da avaliação, mas também a sua aderência às necessidades da população. Ao escolher os indicadores de resultado sobre o nível de saúde da população, ao invés dos indicadores de processo, o avaliador estará contemplando a atenção às necessidades de saúde e não a oferta de serviços de saúde.

$\mathrm{Na}$ avaliação, o julgamento de valor ocorre no momento em que é feita uma comparação entre o conhecimento obtido do objeto e os valores de referência estabelecidos. Há uma interdependência, portanto, entre um indicador e o seu respectivo parâmetro de forma que a inexistência de um invalida a utilização do outro. A escolha prévia do parâmetro direcionará o detalhamento necessário para a construção do indicador, pois per- 
mitirá que a seleção de indicadores tenha a abrangência necessária para a emissão do juízo de valor ${ }^{8}$.

Na AGSS os parâmetros são índices quantitativos ou qualitativos estabelecidos como referência para serem alcançados com os esforços empreendidos para esse fim. Eles devem ser estabelecidos nas condições reais em que as ações são desenvolvidas. Um parâmetro se diferencia, portanto, de um padrão pelo fato de que este se refere a um índice estabelecido em condições ideais. A utilização de padrões como parâmetros trazem vieses na avaliação dos serviços de saúde, pois, considerando que condições ideais no campo da saúde são raras, essa utilização poderia conduzir a juízos de valor desfavoráveis a uma adequada tomada de decisão.

Assim como para os indicadores, o estabelecimento de parâmetros também constitui uma atividade crítica, pois o julgamento de valor emitido na avaliação depende de sua adequada instauração. Entende-se por adequado aquele parâmetro que: tenha correspondência com o indicador escolhido; considere os recursos disponíveis e/ou passíveis de serem mobilizados; seja aceito pelos gestores, técnicos e por outros interessados na avaliação; e seja factível de ser atingido com os esforços empreendidos para esse fim no contexto real em que a ação é desenvolvida. É o parâmetro que permite comparar os resultados obtidos e que assegura a distinção essencial entre a avaliação e a mera análise de situação. Se a definição do parâmetro se dá depois da utilização dos indicadores, isto se constitui apenas numa análise em que procura entender o que foi observado através dos indicadores.

$\mathrm{Na}$ gestão de serviços de saúde os parâmetros mais comumente utilizados são aqueles estabelecidos como objetivos ou metas nos planos, nos programas ou nos pactos. As áreas técnicas de organismos internacionais, nacionais, estaduais, municipais, os órgãos representativos de classe e de especialidades da área da saúde também constituem importantes fontes de parâmetros utilizáveis para a avaliação.

\section{Abordagem e técnicas de pesquisa}

O processo avaliativo deve ser construído em bases lógicas, coerentes e racionais, utilizando mecanismos e instrumentos de pesquisa existentes de forma a garantir a sua consistência e validade, e dar confiabilidade aos atores envolvidos na utilização dos seus resultados e na implementação da tomada de decisão.

Apesar da permanente busca de dar racionalidade e cientificidade ao processo de avaliação, ela deve ser realizada dentro do limite de tempo disponível ou exigido para a sua utilização, ou seja, A avaliação deve fornecer as respostas aos tomadores de decisão em tempo para que elas sejam levadas em conta em suas decisões. Uma informação perfeita de uma avaliação ideal é inútil se ela chega depois que a decisão já tenha sido tomada, uma situação muito frequente ${ }^{3}$.

Nessas condições, deve-se buscar a utilização de pressupostos, metodologias e instrumentos os mais apropriados aos fins da avaliação, ou seja, utilizar desenhos relativamente abertos e especificos para cada tipo de aproximação e apreensão da realidade buscada ${ }^{15}$ e utilizar níveis de confiança mais flexíveis, mas que sejam suficientes para trazer melhorias na tomada de decisão. Nesse último aspecto, Habicht et al. são enfáticos: Em qualquer situação o sacrossanto limite de significância de $5 \%$ precisa ser questionado antes de ser aceito automaticamente. [...]. Explicitar os niveis do percentual de erro que são apropriados para o tomador de decisão é, de fato, mais científico do que ir aceitando cegamente os niveis convencionais ${ }^{3}$.

A utilização de dados secundários para a AGSS, particularmente os proporcionados pelas bases de dados sobre informações de saúde existentes no país, constitui uma vertente importante nos estudos avaliativos. Essa alternativa merece ser fortemente encorajada pela disponibilidade de informações, de custo, de rapidez na obtenção de resultados e de potencial de impacto nos serviços de saúde.

A existência de um problema e a necessidade de compreender e buscar soluções para resolvêlo em um contexto multifacetado, multiprofissional e interdisciplinar fornece à AGSS um conjunto de características que, na perspectiva de Creswell ${ }^{16}$, caracterizam um conjunto de alegações de conhecimento pragmático, ou seja: que elas surgem a partir de ações, de situações e de consequências; que há uma preocupação com as aplicações e as soluções aos problemas; que estes são mais importantes que os métodos; e que é necessário utilizar todos os meios para entender o problema. Essas alegações levam à adoção de métodos pluralistas e mistos de pesquisa, que utilizam tanto técnicas quantitativas como qualitativas.

O que torna científica uma avaliação não é a descoberta de uma única verdade, e sim o esforço para verificar observações e validar o seu significado ou seus diferentes significados ${ }^{17}$. Esse aspecto é reafirmado por Tanaka e Melo ao explicitar que a abordagem quantitativa permite revelar aspectos gerais do fenômeno avaliado e a abordagem qualitativa centra-se na explicação de aspectos do mesmo fenômeno ${ }^{10}$ e acrescentam que, conside- 
rando a heterogeneidade e complexidade dos serviços de saúde é aconselhável que o desenho da avaliação utilize uma mescla das abordagens metodológicas qualitativas e quantitativas. Isto porque, ao mesmo tempo em que se complementam, permitem avaliar diferentes facetas de um mesmo fenômeno e, dessa maneira, possibilita uma resposta mais adequada à pergunta avaliativa ${ }^{10}$.

\section{Julgamento de valor, tomada} e implementação de decisão

Ao longo do processo avaliativo são levantados elementos que aumentam o conhecimento do objeto, das suas diversas facetas e do contexto onde ele se situa, no entanto, na leitura e interpretação dos dados (indicadores), há que se cuidar para assegurar coerência de análises contextualizadas, ultrapassando o olhar sobre as estatísticas como resultados finais, isolados, neutros, e dialogando com as interpretações dos sujeitos envolvidos, à luz das lógicas culturais, relações institucionais e estruturas sociais ${ }^{14}$.

A subjetividade presente na avaliação pode ser reduzida, mas jamais eliminada, pois na emissão do julgamento final, além dos dados objetivos obtidos no processo avaliativo, é necessário levar em conta a influência dos valores próprios do avaliador, os seus princípios, crenças e convicções. Como ela não pode ser evitada, não se deve buscar anular ou esconder esta subjetividade, mas torná-la a mais explícita possivel ${ }^{8}$. A clareza do alcance e dos limites contribui para a confiabilidade de uma avaliação.

Os resultados da avaliação têm que responder à questão que o gestor se coloca. Eles não se traduzem automaticamente em uma decisão, mas espera-se que as informações produzidas contribuam para o julgamento de uma determinada situação com maior validade, influenciando positivamente as decisões, para isso ela tem que ser pertinente, ter fundamentação teórica e credibilidade ${ }^{1}$.

A tomada de decisão é o momento em que o gestor recebe os resultados da avaliação e decide sobre as ações a serem empreendidas para solucionar o problema que lhe deu origem. Esse momento também é revestido de subjetividade, pois o gestor tem que contextualizar os resultados da avaliação dentro dos vários cenários e fatores que lhe são próprios: questões estratégicas, políticas, econômicas, circunstanciais (oportunidades), enfim, os diversos fatores que afetam a gestão de um serviço, e formar uma convicção que lhe permitirá tomar a decisão.

Para que o problema seja efetivamente resolvido é necessário que todos os atores envolvidos na execução da decisão realizem a sua parte. A utilização da margem de poder que cada ator possui em favor da concretização da ação constitui uma tarefa que exige sensibilidade, pois é necessário que os seus resultados permitam aos diferentes atores envolvidos, que podem ter campos de julgamento diferentes, se posicionarem e construírem (individual ou coletivamente) um julgamento capaz de se traduzir em ação ${ }^{1}$.

\section{Conclusão}

A AGSS tem um compromisso com a melhoria dos níveis de saúde da população através do desenvolvimento de processos avaliativos participativos que levem em consideração tanto os objetivos dos serviços de saúde quanto as necessidades de saúde da população.

É uma forma de contribuir para a obtenção de resultados concretos na saúde dos usuários dos serviços de saúde, portanto, para subsidiar o processo de tomada de decisão, a avaliação deve ser direcionada tecnicamente para as principais necessidades de saúde, respeitando os princípios constitucionais da política de saúde do país, e de conduzir o seu desenvolvimento de forma a construir a viabilidade da implementação das ações resultantes das decisões tomadas.

Utilidade, oportunidade, factibilidade, confiabilidade, objetividade e direcionalidade constituem o conjunto de princípios que permitem a estruturação de processos avaliativos na lógica da AGSS, ou seja, processos potencialmente capazes de propiciar impactos efetivos na saúde da população.

A dinâmica dos serviços de saúde dificulta a realização de avaliações em função da necessidade de se tomar decisões no momento em que os problemas acontecem ou são identificados. Nesse contexto, para que a AGSS possa atingir os seus objetivos, ela terá que se tornar em uma ação permanente na prática dos serviços.

A incorporação da lógica da avaliação no cotidiano de todos aqueles que têm o poder de decidir e de implementar ações será condição essencial para a institucionalização da avaliação na gestão dos serviços de saúde. No entanto, algumas questões precisam ser exploradas no campo da avaliação para a implementação desta estratégia:

- Qual a possibilidade real de que a avaliação seja utilizada e valorizada como um instrumento racionalizador da tomada de decisão na gestão em saúde?

- Como contemplar na avaliação da gestão as contradições existentes no processo de construção do SUS? 
- Quais os cuidados necessários para que a avaliação da gestão seja concluída no tempo politico da decisão sem que haja perda da confiabilidade das informações apresentadas?

- Será possível a avaliação da gestão com poucos indicadores?

As questões apresentadas merecem um processo de reflexão que possibilite identificar os limites e alcances da presente proposta de avaliação da gestão.

\section{Colaboradores}

OY Tanaka e EM Tamaki participaram igualmente de todas as etapas de elaboração do artigo.

\section{Referências}

1. Contandriopoulos AP. Avaliando a institucionalização da avaliação. Cien Saude Colet 2006; 10(3):705711.

2. Novaes HMD. Pesquisa em, sobre e para os serviços de saúde: panorama internacional e questões para a pesquisa em saúde no Brasil. Cad Saude Publica 2004; 20(Supl. 2):S147-173.

3. Habicht JP, Victora CG, Vaughan JP. Evaluation designs for adequacy, plausibility and probability of public health programme performance and impact. International Journal of Epidemiology 1999; 28(1):10-18.

4. Hartz Z, organizadora. Avaliação em saúde. Rio de Janeiro: Fiocruz; 1997.

5. Matida AH, Camacho LAB. Pesquisa avaliativa e epidemiologia: movimento e síntese no processo de avaliação de programas de saúde. Cad Saude Publica 2004; 20(1):37-47.

6. Contandriopoulos AP, Champagne F, Denis J-L, Pineault R. Avaliação na área da saúde: conceitos e métodos. In: Hartz Z, organizadora. Avaliação em saúde. Rio de Janeiro: Fiocruz; 1997. p. 29-47.

7. Paim JS, Teixeira CF. Política, planejamento e gestão em saúde: balanço do estado da arte. Rev Saude Publica 2006; 40(N.Esp):73-78.

8. Tanaka OY, Melo C. Avaliação de serviços e programas de saúde para a tomada de decisão. In: Rocha AM, Cesar CLM, editores. Saúde pública: bases conceituais. São Paulo: Atheneu; 2008. p. 119-131.

9. Vieira da Silva LM. Conceitos, abordagens e estratégias para a avaliação em saúde. In: Hartz ZMA, Vieira da Silva LM, organizadoras. Avaliação em saúde. Salvador: EDUFBA; 2005. p. 15-39.
10. Tanaka OY, Melo C. Reflexões sobre a avaliação em serviços de saúde e a adoção das abordagens qualitativa e quantitativa. In: Bosi MLM, Mercado FJ, organizadores. Pesquisa qualitativa de serviços de saúde. Petrópolis: Vozes; 2004. p. 121-136.

11. World Health Organization (WHO). Health programme evaluation. Geneva: World Health Organization (WHO); 1981.

12. Rede Interagencial de Informações para a Saúde (RIPSA). Indicadores básicos de saúde no Brasil. Brasília: Organização Pan-americana de Saúde (OPAS); 2002.

13. Tanaka OY, Melo C. Avaliação de programas de saúde do adolescente. São Paulo: Editora da USP; 2001.

14. Santos-Filho SB. Perspectivas da avaliação da Política Nacional de Humanização em Saúde. Cien Saude Colet 2007; 12(4):999-1010.

15. Novaes HMD. Avaliação de programas, serviços e tecnologias em saúde. Rev Saude Publica 2000; 24(5): 547-559.

16. Creswell JW. Projeto de pesquisa: método qualitativo, quantitativo e misto. $2^{\text {a }}$ ed. Porto Alegre: Artmed; 2007.

17. Uchimura KY, Bosi MLM. Qualidade e subjetividade na avaliação de programas e serviços de saúde. Cad Saude Publica 2002; 18(6):1561-1569. 